\title{
Influência do escore de trato reprodutivo e da circunferência escrotal dos touros sobre a antecipação da puberdade em novilhas Nelore
}

DOI: https://doi.org/10.35168/2176-896X.UTP.Tuiuti.2020.Vol7.N62.pp140-165

Welington Hartmann

Graduação em Medicina Veterinária - Universidade Federal do Paraná (1983), Mestrado em Ciências Veterinárias Universidade Federal do Paraná (2002) e Doutorado em Tecnologia de Alimentos pela Universidade Federal do Paraná. (2009). Link Lattes: http://lattes.cnpq.br/1128697528473748 Orcid: 0000-0001-7321-8033 Universidade Tuiuti do Paraná (UTP), Curitiba, PR, Brasil. E-mail: welington.hartmann@utp.br

Hedron Victor da Luz Grosse Graduação em andamento em Medicina Veterinária. Universidade Tuiuti do Paraná, UTP, Brasil. Link Lattes: http://lattes.cnpq.br/0390063439679186 Orcid://orcid.org/0000-0002-9165-619X Universidade Tuiuti do Paraná (UTP), Curitiba, PR, Brasil. 


\section{Influência do escore de trato reprodutivo e da circunferência escrotal dos touros sobre a antecipação da puberdade em novilhas Nelore}

\section{Resumo}

A raça Nelore é considerada tardia sexualmente, pois enquanto fêmeas taurinas atingem a puberdade com cerca de 10 a 15 meses, nos zebuínos ocorre dos 16 a 40 meses. Há uma constante busca constante de técnicas efetivas que melhorem a eficiência reprodutiva. A puberdade é determinada por fatores genéticos, juntamente com a taxa de crescimento e peso corporal, que são bastante influenciados por fatores ambientais, destacando o manejo nutricional. O estudo foi realizado em uma propriedade localizada em Porto Esperidião-MT, com a utilização de 223 fêmeas Nelore PO com idades que variaram de 12 a 15 meses. Um grupo de novilhas foi formado por filhas de touros com circunferência escrotal (CE) de $40 \mathrm{~cm}$, outro grupo por filhas de touros com CE de $41 \mathrm{~cm}$ e assim por diante, até $46 \mathrm{~cm}$, totalizando 7 grupos. O tratamento estatístico R-Quadrado indicou que o modelo ajustado explica 88,1536\% da variabilidade na CE. O coeficiente de correlação foi igual a 0,938902 , indicando uma relação relativamente forte entre as variáveis CE e peso aos 13 meses. O tratamento estatístico permitiu obter um modelo linear para descrever a relação entre Peso $(\mathrm{kg})$ e CE $(\mathrm{cm})$, com a equação: Peso $(\mathrm{kg})=-20,6429+8,5 \times C E(\mathrm{~cm})$. Com essas informações é possível auferir a importância da medida de circunferência escrotal em touros da raça Nelore, por apresentar correlação alta e positiva com o peso corporal de novilhas e com escore de trato reprodutivo aos 13-14 meses de idade.

Palavras-chave: Bovinocultura. Circunferência escrotal. Precocidade. 


\section{Influence of the score of reproductive tract and scrotal circumference of sires on the anticipation of puberty in Nellore heifers}

\section{Abstract}

Nellore breed is considered sexually late, because while taurine females reach puberty at about 10 to 15 months, in zebuins that occurs from 16 to 40 months. There is a constant search for effective techniques that improve reproductive efficiency. Puberty is determined by genetic factors, along with growth rate and body weight, which are greatly influenced by environmental factors, highlighting nutritional management. The study was carried out in a farm located in Porto Esperidião-MT, with the use of 223 Nellore PO females with ages ranging from 12 to 15 months. One group of heifers was formed by daughters of bulls with scrotal circumference (SC) of $40 \mathrm{~cm}$, another group by daughters of bulls with SC of $41 \mathrm{~cm}$ and so on, up to $46 \mathrm{~cm}$, totaling 7 groups. The statistical treatment R-Square indicated that the adjusted model explains $88.1536 \%$ of the variability in SC. The correlation coefficient was equal to 0.938902 , indicating a relatively strong relationship between the variables SC and weight at 13 months. The statistical treatment allowed to obtain a linear model to describe the relationship between weight $(\mathrm{kg})$ and $\mathrm{SC}(\mathrm{cm})$, with the equation: Weight $(\mathrm{kg})=-20.6429+8.5 \mathrm{x}$ SC $(\mathrm{cm})$. With this information, it is possible to assess the importance of scrotal circumference measurement in Nellore bulls, because it presents a high and positive correlation with the body weight of heifers and with reproductive tract score at 13-14 months of age.

Keywords: Cattle. Scrotal circumference. Precocity. 


\section{Influência do escore de trato reprodutivo e da circunferência escrotal dos touros sobre a antecipação da puberdade em novilhas Nelore}

\section{Introdução}

A precocidade sexual tem sido um dos objetivos das áreas técnicas na bovinocultura moderna, pois apresenta vantagens marcantes como: redução da idade ao primeiro parto, redução da idade de abate, aumento da taxa de progresso genético com a redução do intervalo de gerações e diminuição do custo do animal na propriedade, resultando em maior retorno financeiro. Vários fatores estão associados à indução da puberdade em novilhas, como a herança genética, nutrição, fatores de ambiente, e presença de machos (ARAUJO et al., 2019).

A raça Nelore é considerada tardia sexualmente, pois enquanto fêmeas taurinas atingem a puberdade com cerca de 10 a 15 meses, nos zebuínos ocorre dos 16 a 40 meses (HAFEZ e HAFEZ, 2004). Desta forma, existe a busca constante de técnicas efetivas que melhorem a eficiência reprodutiva das fêmeas Nelore, somando-se à qualidade de seu produto final (ARAUJO et al., 2018).

Precocidade sexual se caracteriza pelo aumento antecipado dos pulsos de hormônio luteinizante (LH), nas concentrações de estradiol, no diâmetro de folículos dominantes e na duração das ondas foliculares no período da pré-puberdade. A seleção de novilhas de acordo com seu grau de maturidade sexual pode reduzir os custos de alimentação e aumentar a taxa de concepção no acasalamento (FREITAS, 2005). Ultimamente, diversos protocolos hormonais têm sido utilizados para induzir a puberdade em novilhas. Estes protocolos se baseiam na junção ou não de vários hormônios, salientando o uso dos dispositivos de liberação de progesterona associados ao uso de estradiol para sincronizar a emergência da onda folicular (BÓ et al., 2018). A progesterona é comumente usada na forma de dispositivos intravaginais, aumentando em poucas horas a concentração circulante nos 


\section{Influência do escore de trato reprodutivo e da circunferência escrotal dos touros sobre a antecipação da puberdade em novilhas Nelore}

níveis supraluteais, bloqueando o eixo hipotálamo-hipófise-gonadal, impedindo a pulsatilidade do hormônio luteinizante. Com a remoção do dispositivo de progesterona a manifestação do estro ocorre de 48 a 72 horas (PEREIRA e HARTMANN, 2018).

O objetivo do presente trabalho foi verificar a correlação entre as variáveis circunferência escrotal (CE) e peso da progênie aos 13 meses com a finalidade de aumentar a taxa de prenhez aos 14 meses (PP14) em rebanhos da raça Nelore na região do Mato Grosso.

\section{Revisão de Literatura}

\subsection{Ciclo estral}

A compreensão detalhada dos eventos que ocorrem durante o ciclo estral da fêmea bovina é de extrema importância para a manipulação do mesmo (NICIURA, 2015).

As vacas são uma espécie poliéstrica contínua, ou seja, os ciclos éstricos ocorrem ao longo do ano até serem interrompidos pela gestação. O ciclo estral dos bovinos tem uma duração média de três semanas, mas pode variar entre 17 e 25 dias (FORTES et al., 2018).

Para facilitar o entendimento do ciclo estral da fêmea bovina, pode-se separá-lo em 4 fases: proestro, estro, metaestro e diestro (FERREIRA et al., 2010).

O proestro começa quando as concentrações de progesterona do ciclo anterior baixam para níveis basais devido à regressão do CL; e termina quando o comportamento de receptividade sexual começa. É caracterizado pelo crescimento do folículo dominante da última onda folicular 


\section{Influência do escore de trato reprodutivo e da circunferência escrotal dos touros sobre a antecipação da puberdade em novilhas Nelore}

do ciclo anterior; portanto, sua duração depende do grau de desenvolvimento em que o folículo se encontra no momento da luteólise (SILVA, 2020). Tem duração média de 2 a 3 dias, terminando com a aceitação do macho, o que caracteriza a iniciação da próxima fase.

O período denominado estro é caracterizado pela manifestação do cio, ou seja, a fêmea apresenta sinais de receptividade sexual e tem seu fim com a ovulação (NICIURA, 2015). Neste período, observamos elevados níveis de estrógeno circulante, fazendo com que o útero esteja túrgido, a cérvix relaxada, vagina e vulva edemaciadas e hiperêmicas. As fêmeas bovinas apresentam manifestações comportamentais de estro, como imobilidade durante a monta, comportamento homossexual, descarga de muco vaginal, vocalização frequente, inquietação, aumento da frequência de micção, entre outras, influenciadas pela idade, condições ambientais e fatores sociais como a hierarquia (MELLO, 2019). É uma fase de curta duração podendo variar de 5 a 21 horas, sendo em média de 13,4 horas (FERREIRA et al., 2010). Os bovinos em estro são caracterizados por alterações comportamentais e morfológicas, podendo-se evidenciar inquietação, vocalização excessiva, inapetência, vulva edemaciada e brilhante, secreção vaginal, comportamento homossexual de montar e, principalmente, deixar ser montada por vacas e touros, demonstrando assim, a receptividade para a realização da cópula (LOIOLA, 2016).

O metaestro das fêmeas bovinas é caracterizado como o período entre o final do cio até o $5^{\circ}$ dia do ciclo estral. É nesse dia que ocorre a ovulação, de 24 a 48 horas após o início do cio. Fase de percepção mais difícil, com duração de aproximadamente 2 a 4 dias e corresponde à formação do CL (FURTADO et al., 2011). Com a produção inicial de progesterona pelo CL, os órgãos genitais tendem a ficar com menor tônus e menos vascularizados. 


\section{Influência do escore de trato reprodutivo e da circunferência escrotal dos touros sobre a antecipação da puberdade em novilhas Nelore}

No diestro é verificado o total desenvolvimento do corpo lúteo e os órgãos reprodutivos ficam sob domínio da progesterona. As glândulas uterinas se atrofiam e a cérvix se encontra fechada e as mucosas genitais pálidas e secas. Nas fêmeas vazias, o diestro dura de 13 a 16 dias, indo do $4^{\circ}$ ao $17^{\circ}$ dia do ciclo. Caso o óvulo não seja fecundado, em torno do $16^{\circ}$ dia, o CL inicia um processo de regressão em decorrência da ação da PGF2 $\alpha$ secretada pelo útero e o diestro é seguido pelo proestro do ciclo seguinte, reiniciando o processo (PEREIRA e HARTMANN, 2018).

Quanto à fisiologia hormonal, o ciclo estral pode ser caracterizado por duas fases. A folicular corresponde ao período de elevada secreção de estradiol (NICIURA, 2015). Essa vai da regressão do CL à ovulação, tendo o folículo dominante com a estrutura característica predominante e compreende $20 \%$ da duração do ciclo (FERREIRA et al., 2010). E a fase luteínica é aquela em que o CL é a estrutura dominante, por isso há elevada secreção de progesterona (NICIURA, 2015). Essa corresponde a $80 \%$ da duração do ciclo estral e vai da ovulação até a regressão do CL (FERREIRA et al., 2010). Os períodos de proestro e estro compõem a fase folicular, enquanto o diestro ocorre na fase luteínica (NICIURA, 2015).

\subsection{Precocidade em fêmeas bovinas}

O rendimento reprodutivo está interligado ao início da vida produtiva das fêmeas, chocando o intervalo entre gerações, a intensidade de seleção e, consequentemente, a taxa de ganho genético do rebanho. A escolha de novilhas mais precoces sexualmente é uma prática adotada por alguns programas de melhoramento genético animal (FORTES et al., 2012). 


\section{Influência do escore de trato reprodutivo e da circunferência escrotal dos touros sobre a antecipação da puberdade em novilhas Nelore}

A categoria que mais impõe limites ao retorno econômico são as novilhas, pelo fato de que elas normalmente demoram de 22 a 36 meses para terem a primeira ovulação e de 44 a 48 meses para produzirem o primeiro bezerro. A idade a primeira cria é um dos fatores que afeta diretamente a determinação da eficiência reprodutiva, além de proporcionar maiores lucros para a redução de custos de manutenção das novilhas (BARUSELLI et al., 2014).

A puberdade é determinada por fatores genéticos, juntamente com a taxa de crescimento e peso corporal, que são bastante influenciados por fatores ambientais, destacando o manejo nutricional. Durante a fase que antecede a entrada na reprodução, maiores ganhos de peso proporcionam maior e mais rápido desenvolvimento do aparelho reprodutivo, levando à antecipação da puberdade (BRUNES, 2017).

Apesar da diferença existente na definição de puberdade entre sexos, existem medidas mensuradas no macho favoravelmente associadas à precocidade sexual em novilhas, com destaque para a circunferência escrotal (CE), que vem sendo amplamente utilizada como indicador de características reprodutivas nas fêmeas (SANTANA JR. et al., 2011). Sollecito et al. (2016) relatam que a precocidade reprodutiva está relacionada com a velocidade do crescimento da fêmea, uma vez que a puberdade é determinada principalmente por aspectos genéticos e nutricionais.

Sob a premissa de que a eficiência reprodutiva dos rebanhos é fundamental para o crescimento da pecuária sustentável, procura-se alcançar a maior quantidade de bezerros nascidos das fêmeas aptas à reprodução. Assim procura-se a eficiência reprodutiva, mensurada pelo aumento da quantidade e qualidade dos bezerros produzidos. 


\section{Influência do escore de trato reprodutivo e da circunferência escrotal dos touros sobre a antecipação da puberdade em novilhas Nelore}

Neste sentido os programas de inseminação artificial em tempo fixo (IATF) se consolidam como ferramenta estratégica de manejo capaz de promover tanto o aumento quantitativo quanto qualitativo da produção de uma propriedade de cria. Desta forma, as matrizes pertencentes ao manejo reprodutivo são o grande desafio para o incremento dos índices, se tornando o foco principal de atenção do sistema produtivo (GALVÃO et al., 2020).

Para a mensuração da eficiência reprodutiva nos rebanhos, uma importante característica reprodutiva é a probabilidade de prenhez da novilha aos 14 meses de idade (PP14), de fácil mensuração e que apresenta herdabilidade média $\left(h^{2}=0,57\right)$, segundo Eler et al. (2002).

\subsection{Circunferência escrotal ou perímetro escrotal}

As características biométricas podem auxiliar na previsão da puberdade dos descendentes, uma vez que testículos maiores em touros jovens estão associados com maturidade sexual precoce (BARTH e OMINSKI, 2000). Medidas de CE têm sido usadas devido a associação com a capacidade reprodutiva de touros e matrizes (BUZANSKAS et al., 2017). Entre os caracteres reprodutivos, aqueles ligados a precocidade reprodutiva são os que contribuem com maior ganho genético, uma vez que antecipam a idade média dos pais no nascimento da sua progênie, ou seja, contribuem para diminuir o intervalo de gerações, principalmente devido ao elevado intervalo de gerações dos bovinos (CARVALHO, 2017).

A CE está correlacionada geneticamente com a idade à puberdade tanto nos machos quanto nas fêmeas, com a fertilidade dos machos e das fêmeas apresentadas a estes machos (CIRYLLO et al., 2001). Pereira et al. (2002) demonstraram que a CE pode ser utilizada como critério de seleção 


\section{Influência do escore de trato reprodutivo e da circunferência escrotal dos touros sobre a antecipação da puberdade em novilhas Nelore}

para melhorar a precocidade sexual das fêmeas, uma vez que a correlação genética indica que seleção de touros com base no mérito genético da CE pode resultar na diminuição da idade ao primeiro parto de suas filhas.

Os estudos associando CE com a idade ao primeiro parto em rebanhos da raça Nelore mostram correlações genéticas favoráveis entre essas características (TEREKADO, 2011).

\subsection{Exame andrológico e congelamento de sêmen}

Os touros que possuem alto valor genético e que demonstram características raciais desejáveis, são selecionados para que seja realizada a coleta e congelamento de seu sêmen. O processo consiste na avaliação da circunferência escrotal que está relacionada com a fertilidade reprodutiva, formato, simetria, consistência do testículo, cauda do epidídimo e palpação transretal para avaliação das glândulas anexas. Para a coleta é utilizado um eletro ejaculador por via retal, que libera pequenas ondas elétricas, estimulando o touro e resultando na ejaculação. Imediatamente após a coleta avaliase motilidade, vigor, turbilhonamento e concentração. Se for aprovado, passa para a etapa do congelamento. Posterior ao congelamento ainda se faz uma última análise para saber se ele está apto a ser utilizado, observando-se motilidade, turbilhonamento e ausência de defeitos.

\subsection{Avaliação do Escore de Trato Reprodutivo (ETR)}

O ETR é um método prático que pode ser utilizado como auxiliar na seleção de novilhas de corte. A classificação das novilhas de acordo com seu grau de maturidade sexual pode reduzir os custos com alimentação e aumentar a taxa de concepção no acasalamento. 


\section{Influência do escore de trato reprodutivo e da circunferência escrotal dos touros sobre a antecipação da puberdade em novilhas Nelore}

Madureira e Pimentel (2005) sugerem a avaliação do escore de trato reprodutivo (ETR) por palpação retal no momento da colocação do implante intravaginal.

Segundo Rosenkrans e Hardin (2003) a avaliação do ETR realizada previamente à temporada reprodutiva evita que animais com baixo potencial reprodutivo sejam utilizados, o que poderia acarretar maiores custos com manejo da reprodução.

O ETR 1 é atribuído a novilhas com útero e ovários pequenos desprovidos de estruturas significativas, sem apresentar tônus, novilhas com ETR 1 provavelmente estão mais distantes da puberdade, se caracterizam pela baixa

frequência de pulsos de $\mathrm{LH}$, devido ao fato de que o hipotálamo e a hipófise ainda são altamente sensíveis ao feedback negativo do estrogênio. Novilhas com ETR 2 estão mais perto da puberdade que aquelas de ETR 1, devido principalmente aos maiores cornos uterinos e ovários. Novilhas com ETR 3 são consideradas muito próximas à ciclicidade ovariana devido ao maior tônus uterino e folículos palpáveis. Pontuações de trato reprodutivo 2 e 3 são associados com a peri puberdade, fase que é caracterizada pela diminuição do feedback negativo de estrogênio, provocando aumentos na frequência de pulsos de LH, crescimento folicular e secreção de estradiol. O declínio do feedback negativo de estrogênio e aumento da secreção de LH resulta em aumento significativo no crescimento folicular e elevada concentração de estradiol, sendo suficiente para induzir o estro e o pico pré-ovulatório de LH. Novilhas com ETR 4 são consideradas cíclicas, indicado pelo tônus e tamanho do útero, consolidação da curvatura dos cornos uterinos e presença de um folículo pré-ovulatório (FRANÇA, 2016). 


\section{Influência do escore de trato reprodutivo e da circunferência escrotal dos touros sobre a antecipação da puberdade em novilhas Nelore}

\subsection{Acetato de Melengestrol (MGA)}

A molécula de MGA é um esteróide progestacional sintético de atividade oral, a partir da adição de um radical metil a medroxiprogesterona (MAP). Foi usado inicialmente em confinamentos de novilhas como agente de crescimento, aumentando a efetividade e ganho de peso dos animais (LOIOLA, 2016).

O seu uso é recomendado para melhorias na fixação e taxas de concepção de vacas em anestro pós-parto, por auxiliar na sincronia do estro e indução da ovulação (WOOD-FOLLIS et al., 2004). Loiola (2016) relata que novilhas tratadas com MGA por oito dias tiveram aumento nos pulsos de LH e todas se tornaram púberes ao término do tratamento, enquanto no grupo controle não houve mudança na liberação do LH e na indução da puberdade durante o período analisado.

MGA tem sido muito utilizado para a manutenção de gestação em fêmeas bovinas, devido a sua ação muito próxima a P4. Essa característica foi avaliada em novilhas leiteiras ovariectomizadas bilateralmente aos 56 dias de gestação, onde o MGA na dose de $4 \mathrm{mg} /$ dia foi eficiente em manter a gestação em 86\% dos animais suplementados até os 270 dias de gestação (LOIOLA, 2016).

As principais vantagens de utilizar MGA consistem na fácil administração do produto e o custo relativamente baixo (PEREZ, 2005). Essas características atraem conceitos para utilização do MGA após a inseminação, com a finalidade de melhorar o ambiente uterino e diminuir as perdas embrionárias, entregando maiores índices destes animais em protocolos de IATF (SILVA JÚNIOR et al., 2014). Entretanto, em relação à utilização para esta finalidade, a literatura é escassa. 


\section{Influência do escore de trato reprodutivo e da circunferência escrotal dos touros sobre a antecipação da puberdade em novilhas Nelore}

\subsection{Cipionato de Estradiol}

Os estrógenos são responsáveis por algumas funções na fisiologia reprodutiva, como a indução do estro, características sexuais secundárias, indução da liberação do hormônio liberador de gonadotrofinas (GnRH) e do hormônio luteinizante (LH) (PEREIRA e HARTMANN, 2018).

O estradiol e seus ésteres, são repetidamente utilizados para promover o mando farmacológico do ciclo estral, pois que na ausência de progesterona sua administração pode estimular a liberação de GnRH e LH e causar a ovulação do folículo dominante (ANDRADE et al., 2012).

O cipionato de estradiol, como indutor da ovulação, permite a realização de protocolos de IATF com apenas três manejos, resultando então na minimização do estresse do animal e reduzindo a mão de obra, continuando biodisponível de forma sistêmica por maior tempo, e quando administrado juntamente com a retirada do implante de progesterona, mantêm níveis plasmáticos suficientes para estimular o pico de LH após a queda dos níveis de progesterona (P4) (PALHÃO et al., 2014). O papel do estradiol nos protocolos depende do tempo em que é aplicado, agindo como na atresia folicular, associada a altas concentrações plasmáticas de progesterona ou como indutor de estro e ovulação, associado a baixas concentrações plasmáticas de progesterona. De acordo com o momento da aplicação, ele executará uma ou outra função. Para demonstrar o efeito da atresia folicular da onda em desenvolvimento no ovário da fêmea, o análogo do estradiol deve ser associado ao progestogênio. Quando usado como estimulador da liberação do pico de LH, deve ser aplicado no momento ou após a remoção do análogo de progesterona e luteólise do corpo lúteo presente no ovário. O tempo de ação do cipionato de estradiol (48-72 horas) está associado ao número de tratamentos adotados nos protocolos 


\section{Influência do escore de trato reprodutivo e da circunferência escrotal dos touros sobre a antecipação da puberdade em novilhas Nelore}

devido ao intervalo entre a aplicação do análogo e a liberação do pico de LH (PEREIRA e HARTMANN, 2018).

Programas de sincronização e indução da ovulação já vêm sendo utilizados normalmente em novilhas mestiças (Bos taurus indicus x Bos taurus taurus); Bos taurus taurus (COLAZO et al., 2003) e Bos taurus indicus (BARUSELLI et al., 2004).

\section{Material e Métodos}

O estudo foi realizado em uma propriedade localizada em Porto Esperidião-MT, com a utilização de 255 fêmeas Nelore PO com idades que variaram de 12 a 15 meses.

Essas fêmeas eram filhas de vacas já desafiadas à precocidade e carregavam uma genética positiva a esse critério avaliativo e, para agregar ainda mais uma linhagem positiva para precocidade foram inseminadas com touros que apresentavam DEP's (Diferença Esperada de Progênie) correlacionadas à reprodução. $\mathrm{O}$ valor de uma DEP é dado pela diferença na performance que é esperada para a futura progênie de um animal comparado à performance da futura progênie de todos os outros animais avaliados na análise, quando acasalados a animais de mesmo valor.

Essas novilhas logo após a desmama entraram em um manejo especial relacionado à alimentação, recebendo ração concentrada, visando o ganho de peso para chegarem na estação com 60 a 70\% do seu peso adulto.

A indução foi realizada com a utilização de acetato de melengestrol e cipionato de estradiol, de 5 a 16 de outubro de 2020, totalizando 12 dias. Receberam 2,3 gramas de MGA por animal, 


\section{Influência do escore de trato reprodutivo e da circunferência escrotal dos touros sobre a antecipação da puberdade em novilhas Nelore}

misturado ao concentrado. No dia 17/10/2020 esses animais foram levados ao curral onde receberam uma dose de 0,3 mL de ECP via intramuscular. Entre os dias 18 a 28/10/2020 não foram manejadas e nem receberam o MGA. No dia 29/10/2020 teve início a avaliação do trato reprodutor, com critérios avaliativos para analisar quais novilhas foram responsivas ou não à indução. Foram avaliadas com notas de 1 a 4, sendo respectivamente, 1: animais não responsivos à indução e 4: animais que obtiveram uma boa resposta à indução hormonal. Cada novilha, ao término da avaliação do ETR via ultrassonografia, foi exposta a um protocolo de IATF de 4 manejos: no D0 receberam um implante intravaginal de P4 contendo $1 \mathrm{mg}$ de progesterona, mais a aplicação de $2 \mathrm{~mL}$ de benzoato de estradiol. Do dia 30/10 a 04/11/2020 tiveram um período sem manejo, e no dia 05/11 receberam uma dose de 2,5 $\mathrm{mL}$ de PGF2 $\alpha$. No dia 6 permaneceram em descanso, e no dia 07/11 foi retirado o implante intravaginal, associado a 2,5 mL de PGF $2 \alpha$, e $0,3 \mathrm{~mL}$ de cipionato de estradiol e $1 \mathrm{~mL}$ de ECG. No dia 08/11/2020 permaneceram em descanso para no dia seguinte serem inseminadas de acordo com as indicações de touros que foi realizada anteriormente.

O escore de trato reprodutivo para avaliação de novilhas precoces é classificado da seguinte forma, de acordo com França (2016).

ETR 1: cornos uterinos imaturos, menor que $20 \mathrm{~mm}$ de diâmetro, ovários pequenos (tamanho de ervilha), sem folículos palpáveis.

ETR 2: cornos uterinos de 20-25 mm, sem tônus ou leve tônus uterino, ovários pequenos (tamanho de azeitona), folículos terciários de 8-10 $\mathrm{mm}$. 


\section{Influência do escore de trato reprodutivo e da circunferência escrotal dos touros sobre a antecipação da puberdade em novilhas Nelore}

ETR 3: cornos uterinos de aproximadamente $30 \mathrm{~mm}$, bom tônus uterino, ovários médios (tamanho de ovo de codorna), presença de folículo dominante, folículos maiores que $10 \mathrm{~mm}$.

ETR 4: cornos uterinos maduros, maiores que $30 \mathrm{~mm}$, bom tônus uterino, ovários médios (tamanho de ovo de codorna) e presença de corpo lúteo.

Para o tratamento estatístico dos dados foi utilizada a planilha Excell for Windows, permitindo as estimativas dos coeficientes de correlação entre CE e peso corporal, bem como entre CE e ETR. Também foram acrescentadas as linhas de tendência no estudo sobre CE e ETR, permitindo auferir informações a partir da fórmula obtida. Para a estimativa dos coeficientes de correlação entre CE e peso corporal foi utilizado o tratamento estatístico R-Quadrado (ANOVA) do programa computacional Statgraphics Centurion $\mathbb{R}$. Para esse trabalho foram utilizadas as pesagens de 255 novilhas.

\section{Resultados}

Um grupo de novilhas foi formado por filhas de touros com circunferência escrotal de $40 \mathrm{~cm}$, outro grupo por filhas de touros com CE de $41 \mathrm{~cm}$ e assim por diante, até $46 \mathrm{~cm}$, totalizando 7 grupos. No que se refere ao estudo comparativo entre CE e seu efeito sobre o peso das novilhas à idade de um ano, os resultados podem ser observados na Figura 1. 


\section{Influência do escore de trato reprodutivo e da circunferência escrotal dos touros sobre a antecipação da puberdade em novilhas Nelore}

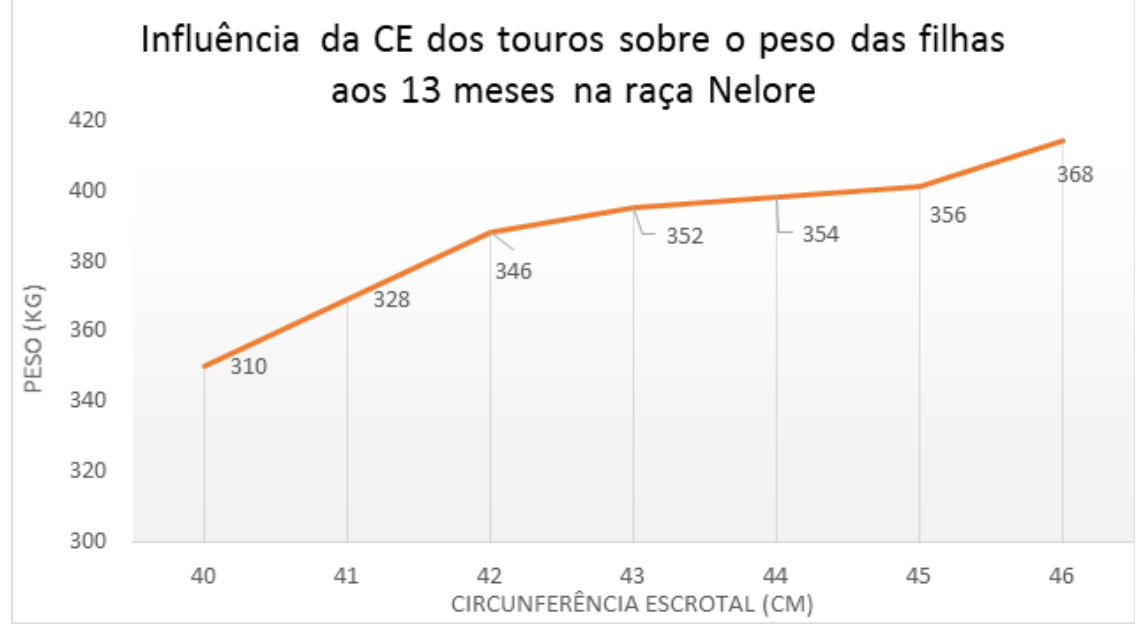

Figura 1: Influência da Circunferência Escrotal dos touros sobre o peso de suas filhas aos 13 meses de idade $(n=243)$

Observa-se um comportamento linear da curva, que se inicia com o grupo de novilhas filhas de touros com CE $40 \mathrm{~cm}$ e que obtiveram peso médio de $310 \mathrm{~kg}$ aos 13 meses de idade. As novilhas foram agrupadas de acordo com a CE dos seus touros progenitores. No grupo CE 40 havia 12 novilhas; no CE 41, 39; no CE 42, 14; no CE 43, 52; no CE 44, 75; no CE 45, 29 e no CE 46, 22; totalizando 243 novilhas. O tratamento estatístico R-Quadrado indica que o modelo ajustado explica $88,1536 \%$ da variabilidade na Circunferência Escrotal (cm). O 


\section{Influência do escore de trato reprodutivo e da circunferência escrotal dos touros sobre a antecipação da puberdade em novilhas Nelore}

coeficiente de correlação é igual a 0,938902 , indicando uma relação relativamente forte entre as variáveis $\mathrm{CE}$ e peso aos 13 meses. O erro padrão da estimativa mostra o desvio padrão dos resíduos como sendo 0,814492 . Este valor pode ser usado para construir limites de previsão para novas observações. Como o valor P na tabela ANOVA é menor que 0,05, existe uma relação estatisticamente significativa entre Circunferência Escrotal $(\mathrm{cm})$ e Peso $(\mathrm{kg})$ no nível de confiança de $95,0 \%$.

O tratamento estatístico permitiu obter um modelo linear para descrever a relação entre Peso (kg) e Circunferência Escrotal (cm). A equação do modelo ajustado é:

$$
\text { Peso }(\mathrm{kg})=-20,6429+8,5 \times \mathrm{CE}(\mathrm{cm})
$$

A Análise de Variância pode ser observada na Tabela 1:

\begin{tabular}{|c|c|c|c|c|c|}
\hline \multicolumn{7}{|c|}{ Análise de Variância } \\
\hline Fonte & $\begin{array}{c}\text { Somados } \\
\text { Quadrados }\end{array}$ & $\begin{array}{c}\text { Graus de } \\
\text { Liberdade }\end{array}$ & $\begin{array}{c}\text { Média dos } \\
\text { Quadrados }\end{array}$ & F-Ratio & Valor deP \\
\hline Modelo & 24,683 & 1 & 24,683 & 37,21 & 0,0017 \\
\hline Residual & 3,31698 & 5 & 0,663396 & - & - \\
\hline Total (Corr.) & 28,0 & 6 & - & - & - \\
\hline
\end{tabular}




\section{Influência do escore de trato reprodutivo e da circunferência escrotal dos touros sobre a antecipação da puberdade em novilhas Nelore}

Com essas informações é possível auferir a importância da medida de circunferência escrotal em touros da raça Nelore, por apresentar correlação alta e positiva com o peso corporal de novilhas e com escore de trato reprodutivo aos 13-14 meses de idade. Esse fato representa uma importante contribuição à seleção genética de touros, que resulta em precocidade sexual de suas filhas. Assim elas passam a contribuir economicamente para o êxito da fazenda, e chegam à idade de 23 ou 24 meses com uma cria ao pé.

$\mathrm{Na}$ continuidade desse estudo, apreciando-se as informações disponibilizadas, as novilhas foram agrupadas de acordo com o ETR, obtido por exame ultrassonográfico antes da IATF. Assim o grupo 1 foi composto por novilhas com cornos uterinos com diâmetros menores que $29 \mathrm{~mm}$ e o grupo 2 acima de $30 \mathrm{~mm}$. Após o agrupamento das informações, calculou-se a porcentagem de novilhas nos dois grupos, de acordo com a CE dos pais. Ao se observar a Figura 2, o grupo ETR1 + ETR2 demonstrou declínio a partir de CE 42 até CE 45, obtendose a fórmula da linha de tendência: $y=-5,55 x+33,9$. Por outro lado, o grupo ETR3 + ETR4 passou a apresentar valores ascendentes em porcentagens de novilhas nos melhores escores de ETR, de acordo com a PE dos touros em ordem crescente. A fórmula obtida pela linha de tendência desse segundo grupo foi estimada: $y=5,55 x+66,1$. A correlação entre CE e ETR foi alta e positiva $(r=0,92283)$. 


\section{Influência do escore de trato reprodutivo e da circunferência escrotal dos touros sobre a antecipação da puberdade em novilhas Nelore}

Influência da CE dos touros sobre o ETR das filhas aos 13 meses

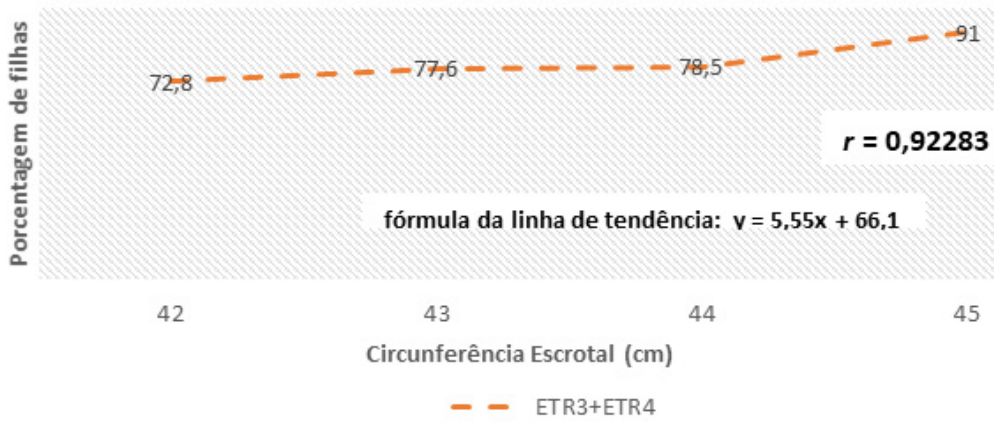

Figura 2: Influência da Circunferência Escrotal dos touros sobre o Escore de Trato Reprodutivo das filhas aos 13 meses de idade $(n=243)$.

As fêmeas que foram expostas ao estudo, com ETR 2, 3 e 4 obtiveram resultados positivos. Das 255 novilhas, 22 (8,6\%) apresentaram ETR 1, e não foram responsivas ao protocolo; 30 novilhas (11,7\%) apresentaram ETR 2, e apresentaram leve resposta ao protocolo; 99 (38,8\%) apresentaram ETR 3, e apresentaram uma boa resposta ao protocolo; e 104 novilhas (40,7\%) apresentaram ETR 4 e apresentaram uma excelente resposta ao protocolo hormonal. 


\section{Influência do escore de trato reprodutivo e da circunferência escrotal dos touros sobre a antecipação da puberdade em novilhas Nelore}

\section{Conclusão}

Diversas características ligadas à eficiência reprodutiva são consideradas nos programas de avaliação genética de bovinos de corte no Brasil. A maioria dessas características, entretanto, apresenta baixa herdabilidade, indicando a necessidade de muito esforço para se obter progresso genético. A indução à puberdade gera maior produção, levando-se em consideração que essas fêmeas vão produzir uma cria a mais que as demais não expostas à indução; também há contribuição à qualidade, devido à contribuição à Diferença Esperada de Progênie dos touros. É necessário avaliar os efeitos da concepção em idade jovem sobre os desempenhos reprodutivo e produtivo futuros das fêmeas, em diferentes sistemas de produção, para tanto utiliza-se o escore do trato reprodutivo, que permite detectar animais que foram e os que não foram responsivos à indução. A circunferência escrotal demonstra constituir um bom critério de seleção, como característica indicadora de precocidade reprodutiva na progênie, e a probabilidade de prenhez aos 14 meses de idade é um critério complementar, ligado diretamente à precocidade reprodutiva das fêmeas.

\section{Referências}

ANDRADE, B.H.A.; FERRAZ, P.A.; RODRIGUES, A.S. Eficiência do cipionato de estradiol e do benzoato de estradiol em protocolos de indução da ovulação sobre a dinâmica ovariana e taxa de concepção de fêmeas nelore inseminadas em diferentes momentos. Arch Vet Sci. 2012; 17:70-82. 


\section{Influência do escore de trato reprodutivo e da circunferência escrotal dos touros sobre a antecipação da puberdade em novilhas Nelore}

ARAUJO, A. C. C.; NONATO, M. S.; BEZERRA, A. R. A. Induction of ovulation in heifers with memorandum of cyclicality. Brazilian Journal of Development, v. 5, n. 11, p. 2428624290, 2019.

ARAUJO, A. C. R.; SALES, A. F. F.; FERREIRA, J. P. V.; NEVES NETO, J. T. Indução à puberdade em novilhas. In: Colóquio Estadual de Pesquisa Interdisciplinar e Congresso Nacional de Pesquisa Multidisciplinar, II-I. Anais... 2018.

BARTH, A. D.; OMINSKI, K. H. The relationship between scrotal circumference at weaning and at one year of age in beef bulls. The Canadian Veterinary Journal, v. 41, n. 7, p. 541-546, jul. 2000.

BARUSELLI, P. S.; MARQUES, M.O.; FERREIRA, R.M. Como aumentar a quantidade e a qualidade de bezerros em rebanhos de corte. Revista Brasileira da Zootecnia, v. 29, n. 5 , p. 1327-1331, 2014.

BARUSELLI, P. S.; REIS, E. L.; MARQUES, M. O. The use of hormonal treatments to improve reproductive performance of anestrusbeef catlle in tropical climates. Anim Reprod Sci. 2004; 82; 486-97.

BÓ, G. A. Programs for fixed-time artificial insemination in South American beef cattle. Animal Reproduction Science, v. 15, n. 1, p. 952-962, 2018. DOI: http://dx.doi. org/10.21451/1984-3143-AR2018-0025

BRUNES, L.C. Estudo genetico-quantitativo de características de crescimento, reprodução, carcaça e escores visuais em um rebanho Nelore sob seleção para precocidade sexual. Dissertação (Mestrado em Produção Animal) - Universidade Federal de Goiás (UFG), Goiânia, GO, 2017. 


\section{Influência do escore de trato reprodutivo e da circunferência escrotal dos touros sobre a antecipação da puberdade em novilhas Nelore}

BUZANSKAS, M.E.; PIRES, P.S.; CHUD, T.C.S. Parameter estimates for reproductive and carcass traits in Nelore beef cattle. Theriogenology, v.92, p. 204-209. 2017.

CARVALHO, C. V. D. Avaliação genético quantitativa e genômica para características reprodutivas de bovinos da raça Nelore. Tese de Doutoramento. Universidade Federal da Bahia. 2017.

COLAZO, M. G.; KASTELIC, J. P.; MAPLETOFT, R. J. Effects of estradiol cypionate (ECP) on ovarian folicular dynamics, synchrony of ovulation, and fertility in CIDR-based, fixed-time AL programs in beef heifers. Theriogenology. 2003; 60:855-65.

CYRILLO, J.N.S.G.; RAZOOK, A.G.; FIGUEIREDO, L. A. Estimativa de tendências e parâmetros genéticos do peso padronizado aos 378 dias de idade, medidas corporais e perímetro escrotal de machos Nelore de Sertãozinho, SP. Revista Brasileira de Zootecnia, v.30, n.1, p.56-65, 2001.

ELER, J.P.; SILVA II, J.A.V.; FERRAZ, J.B.S. Genetic evaluation of the probability of pregnancy at 14 months for Nellore heifers. J. Anim. Sci., 80(4):951-954, 2002.

FERREIRA, A. D. M. Reprodução da fêmea bovina: fisiologia aplicada e problemas mais comuns (causas e tratamentos). 1.ed. Juiz de Fora/MG: Editar Editora Associada, 2010. 422 p.

FRANÇA, I. G. Efeito do escore do trato reprodutivo na dinâmica folicular e dopplerfluxometria útero-ovariana em novilhas Nelore (Bos indicus). 2016. $73 \mathrm{f}$. Dissertação (Programa de Pós-Graduação em Ciência Animal) - Universidade Federal do Maranhão, Chapadinha, 2016.

FORTES, M.R.S.; LEHNERT, S.A.; BOLORMAA, S. Finding genes for economically important traits: Brahman cattle puberty. Anim. Prod. Sci., v.52, p.143-50. 2012. 


\section{Influência do escore de trato reprodutivo e da circunferência escrotal dos touros sobre a antecipação da puberdade em novilhas Nelore}

FORTES, C. H. M.; NASCIMENTO, C. A.; GOES, A. A. D. O manejo reprodutivo dos animais da área de produção animal. XXI Seminário interinstitucional de ensino, pesquisa e extensão, p. 1-4, outubro, 2018.

FURTADO, D. A.; TOZZETTI, D. S.; AVANZA, M. F. B.; DIAS, L. G. G. G. Inseminação artificial em tempo fixo em bovinos de corte. Revista Científica Eletrônica de Medicina Veterinária, Garça/SP, v. 16, n. 9, jan. 2011.

HAFEZ, E. S.; HAFEZ, B. Fisiologia da reprodução. 7 ed. São Paulo: Manole, 2004. 513 p.

LOIOLA, M. V. G. Marcadores de fertilidade e associação da suplementação exógena com progesterona a protocolos de IATF em gado de corte. Tese (Doutorado em Ciência Animal nos trópicos) - Escola de Medicina Veterinária e Zootecnia, Universidade Federal da Bahia. Salvador-Bahia, p 129. 2016.

MADUREIRA, E.H.; PIMENTEL, J.R.V. IATF como ferramenta para melhorar a eficiência reprodutiva. Anais... Congresso Brasileiro de Reprodução Animal, 16, Goiânia, GO. Anais: p.1-8, 2005.

MELLO, R. P. Avaliação nas taxas de prenhez de novilhas Nelore e mestiças de diferentes idades, submetidas à IATF, mediante visualização de cio e aplicação de GNRH. TCC (Graduação em Medicina Veterinária) - Centro de Ciências Rurais, Campus Curitibanos da Universidade Federal de Santa Catarina. Curitibanos, p.41. 2019.

NICIURA, S. C. M. Anatomia e fisiologia da reprodução de fêmeas bovinas. Embrapa, 2015. Disponível em: http://www.alice.cnptia.embrapa.br/alice/handle/doc/48249. Acesso em 10.12.2020.

PALHÃO, M. P.; PIEDADE, C. S.; ARAÚJO, H. L. Sincronização folicular e vascularização do folículo dominante em novilhas mestiças tratadas com estradiol. Rev. Bras. Cienc. Vet. 2014; 21:117-21. 


\section{Influência do escore de trato reprodutivo e da circunferência escrotal dos touros sobre a antecipação da puberdade em novilhas Nelore}

PEREIRA, E.; ELER, J. P.; FERRAZ, J. B. S. Análise genética de características reprodutivas na raça Nelore. Pesq Agropec Bras, v.37, p.703-708, 2002.

PEREIRA, J. F. S.; HARTMANN, W. Regulation of the Hypothalamic-Pituitary Gonadal Axis and the Manipulation of the Estrous Cycle of Bovine Females. Chapter 3. Avid Science, 2018.

PEREZ, G. C. Uso do acetato de melengestrol (MGA), associado a PGF $2 \alpha$ e a GnRH ou benzoato de estradiol em protocolos de inseminação artificial em tempo fixo (IATF) para vacas Nelores paridas. 2005. 80 f. Dissertação (Mestrado) - Universidade Estadual de São Paulo. São Paulo.

ROSENKRANS, K. S.; HARDIN, D. K. Repeatability and accuracy of reproductive tract scoring to determine pubertal status in beef heifers. Theriogenology, v.595, p. 10871092, 2003.

SANTANA, M. L.; ELER, J. P.; FERRAZ, J. B.; MATTOS, E. C. Genetic relationship between growth and reproductive traits in Nellore cattle. Animal, v.6, p.565-570, 2011.

SILVA, E. I. C. Fisiologia Clínica do Ciclo Estral de Vacas Leiteiras: Desenvolvimento Folicular, Corpo Lúteo e Etapas do Estro. Belo Jardim: IFPE, 2020.

SILVA JUNIOR, L. S.; FREIRIA, L. B.; ANGREVESSILVA, G. M. Uso do acetato de melengestrol após protocolos de inseminação artificial em tempo fixo em vacas Nelore multíparas. Revista Brasileira de Saúde e Produção Animal, v.15, n.2, p. 425-429, 2014.

SOLLECITO, N. V.; ANDRADE, V. J.; BARBOSA, F. A. Taxa de fertilidade de novilhas de diferentes grupos genéticos com primeiro serviço aos 14 meses de idade. Arq. Bras. Med. Vet. Zootec.,Belo Horizonte , v. 68, n. 2, p. 361-368, 2016. 


\section{Influência do escore de trato reprodutivo e da circunferência escrotal dos touros sobre a antecipação da puberdade em novilhas Nelore}

TERAKADO, A. P. N. Associações genéticas entre perímetro escrotal e características reprodutivas de fêmeas da raça Nelore. (Dissertação) Faculdade de Ciências Agrárias e Veterinárias - Unesp, Jaboticabal-SP, V.1 p.53, 2011.

WOOD-FOLLIS, S.L.; KOJIMA, F.N.; LUCY, M.C.; SMITH, M.F.; PATTERSON, D.J. Estrus synchronization in beef heifers with progestin-based protocols I. Differences in response based on pubertal status at the initiation of treatment. Theriogenology, v.62, n.8, p.1518-1528, 2004.

Submetido em 10 de março de 2021

Aceito em 15 de maio de 2021

Publicado em 8 de julho de 2021 